\title{
Existence of Two Positive Solutions for Two Kinds of Fractional $p$ -Laplacian Equations
}

\author{
Yong Wu ${ }^{1}{ }^{1}$ and Said Taarabti ${ }^{2}$ \\ ${ }^{1}$ School of Tourism Date, Guilin Tourism University, Guilin 541006, China \\ ${ }^{2}$ Laboratory of Systems Engineering and Information Technologies (LISTI), National School of Applied Sciences of Agadir, \\ Ibn Zohr University, Morocco
}

Correspondence should be addressed to Yong Wu; wuyong@gltu.edu.cn

Received 28 January 2021; Revised 5 February 2021; Accepted 6 February 2021; Published 26 February 2021

Academic Editor: Jiabin Zuo

Copyright (c) 2021 Yong Wu and Said Taarabti. This is an open access article distributed under the Creative Commons Attribution License, which permits unrestricted use, distribution, and reproduction in any medium, provided the original work is properly cited.

The aim of this paper is to investigate the existence of two positive solutions to subcritical and critical fractional integro-differential equations driven by a nonlocal operator $\mathscr{L}_{K}^{p}$. Specifically, we get multiple solutions to the following fractional $p$-Laplacian equations with the help of fibering maps and Nehari manifold. $\left\{\begin{array}{ll}(-\Delta)_{p}^{s} u(x)=\lambda u^{q}+u^{r}, & u>0 \text { in } \Omega, \\ u=0, & \text { in } \mathbb{R}^{N} \backslash \Omega .\end{array}\right.$. Our results extend the previous results in some respects.

\section{Introduction}

In this work, we are concerned with the existence of solutions for a nonlocal integro-differential equation

$$
\begin{cases}-\mathscr{L}_{K}^{p} u(x)=\lambda u^{q}+u^{r}, & u>0 \text { in } \Omega, \\ u=0, & \text { in } \mathbb{R}^{N} \backslash \Omega,\end{cases}
$$

where $\Omega$ is a bounded smooth domain in $\mathbb{R}^{n}, n>p s$ with $s$ $\in(0,1), \lambda>0$, the exponents $r$ and $q$ fulfill $0<q<1<r \leq$ $p_{s}^{*}-1$ with the critical fractional Sobolev exponent $p_{s}^{*}=(n p$ $/(n-p s))(n>p s)$, and $\mathscr{L}_{K}^{p}$ is a kind of nonlocal integrodifferential operator defined by:

$$
\begin{aligned}
\mathscr{L}_{K}^{p} u(x)= & 2 \lim _{\varepsilon \longrightarrow 0^{+}} \int_{\mathbb{R}^{N} \backslash B_{\varepsilon}(x)}|u(x)-u(y)|^{p-2}(u(x) \\
& -u(y)) K(x-y) d y,
\end{aligned}
$$

$x \in \mathbb{R}^{N}$, and $K: \mathbb{R}^{N} \backslash\{0\} \longrightarrow(0,+\infty)$ is a measurable function with the following property:

$$
\begin{cases}\gamma K \in L^{1}\left(\mathbb{R}^{N}\right) & \text { where } \gamma(x)=\min \left\{|x|^{p}, 1\right\}, \\ \text { there } & \text { exists a } k_{0}>0 \text { such that, } \\ K(x) \geq k_{0}|x|^{-(N+p s)} & \text { for any } x \in \mathbb{R}^{N} \backslash\{0\}, \\ K(x)=K(-x) & \text { for any } x \in \mathbb{R}^{N} \backslash\{0\} .\end{cases}
$$

In recent years, the existence and multiplicity of solutions of elliptic equations in nonlinear analysis have attracted the attention of many scholars. In particular, problems with regular nolinearities like $u^{q}+\lambda u^{p}, p, q>0$ and singular nonlinearities $u^{-q}+\lambda u^{p}, p, q>0$. At the same time, elliptic problems can be divided into two categories according to their order: integer order and fractional order.

On the one hand, when $s=1$, in [1], the authors considered a class of semilinear problems with singular nonlinearities. Many results on the existence and multiplicity of solutions for singular problems have appeared in the literature 
[2-6]. For example, authors have investigated a singular problem with the kind of critical growth in [6],

$$
-\Delta u=\lambda u^{-q}+u^{2^{*}-1}, \quad u>0 \text { in } \Omega, u=0 \text { on } \partial \Omega,
$$

where $0<q<1$. They obtained the existence of solutions by means of the Nehari manifold method in a suitable range of $\lambda$.

On the other hand, in [7], Mukherjee and Sreenadh considered the following critical fractional Laplace operator equations with a singular nonlinearity

$$
(-\Delta)^{s} u=\lambda a(x) u^{-q}+u^{2_{s}^{*}-1}, \quad u>0 \text { in } \Omega, u=0 \text { in } \mathbb{R}^{n} \backslash \Omega .
$$

They showed the existence and multiplicity of positive solutions with respect to the parameter $\lambda$ for above equation by using variational methods. Furthermore, in [8], they studied a class of critical fractional problems with a lower order perturbation by means of variational and topological methods; precisely, they proved that the number of nontrivial weak solutions is at least twice the multiplicity of the eigenvalue. More details on the critical case of fractional $p$-Laplace equations can be referred to [9]. In subcritical case, the existence of positive solutions to the following quasi-linear problem

$$
\begin{cases}(-\Delta)_{p}^{s} u=\lambda g(x, u)-f(x, u), & x \in \Omega, \\ u=0, & x \in \mathbb{R}^{N} \backslash \Omega,\end{cases}
$$

is studied by means of truncation and comparison techniques in [10]. Zuo et al. [11] investigated a superlinear fractional elliptic equations; the existence of infinity many solutions is obtained by the fountain theorem in subcritical case. Moreover, they also get at least two solutions for a fractional $p$ -Laplace system by the Nehari manifold method in [12]. We will adopt a new technique, considering both subcritical and critical cases in a more general operator context (see (1)).

In order to state our results, let us introduce some notations. The space

$$
\begin{aligned}
X= & \left\{u \mid u: \mathbb{R}^{N} \longrightarrow \mathbb{R}\right. \text { is measurable, } \\
& \left.\left.u\right|_{\Omega} \in L^{p}(\Omega) \text { and }(u(x)-u(y)) \sqrt[p]{K(x-y)} \in L^{p}(Q)\right\},
\end{aligned}
$$

where $Q=\mathbb{R}^{2 N} \backslash(C \Omega \times C \Omega)$ with $C \Omega=\mathbb{R}^{N} \backslash \Omega$. The space $X$ is endowed with the norm,

$$
\|u\|_{X}=\|u\|_{L^{p}(\Omega)}+\left(\int_{Q}|u(x)-u(y)|^{p} K(x-y) d x d y\right)^{1 / p}
$$

and we define the closed linear subspace

$$
X_{0}=\left\{u \in X: u=0 \text { almost everywhere in } \mathbb{R}^{N} \backslash \Omega\right\},
$$

with the norm

$$
\|u\|_{X_{0}}=\left(\int_{Q}|u(x)-u(y)|^{p} K(x-y) d x d y\right)^{1 / p} .
$$

Let

$$
K: \mathbb{R}^{N} \backslash\{0\} \longrightarrow(0,+\infty)
$$

fulfill condition (3). We have that $C_{0}^{\infty}(\Omega) \subset X_{0}$, and $\left(X_{0}\right.$, $\|\cdot\|_{X_{0}}$ ) is a reflexive Banach space (see [13]). Moreover,

$$
\begin{gathered}
X \subset W^{s, p}(\Omega), \\
X_{0} \subset W^{s, p}\left(\mathbb{R}^{N}\right),
\end{gathered}
$$

where $W^{s, p}(\Omega)$ is the usual fractional Sobolev space endowed norm

$$
\|u\|_{W^{s, p}(\Omega)}=\|u\|_{L^{p}(\Omega)}+\left(\int_{\Omega \times \Omega} \frac{|u(x)-u(y)|^{p}}{|x-y|^{n+p s}} d x d y\right)^{1 / p},
$$

and the embedding

$$
X_{0} \hookrightarrow L^{p_{s}^{*}}(\Omega)
$$

$C_{0}(N, p, s)$ such that, for any $v \in X_{0}, 1<k<p_{s}^{*}$

$$
\|v\|_{L^{k}(\Omega)} \leq C_{0}\|v\|_{X_{0}} .
$$

Definition 1 . We say that $u$ is a weak solution of problem (1), if $\mathrm{u}$ fulfills

$$
\begin{aligned}
\int_{Q}|u(x)-u(y)|^{p-2}(u(x)-u(y))(\varphi(x)-\varphi(y)) K(x-y) d x d y \\
\quad=\int_{\Omega}\left(\lambda u_{+}^{q}(x)+u_{+}^{p}(x)\right) \varphi(x) \mathrm{d} x
\end{aligned}
$$

for all $\varphi \in X_{0}$, where $u_{+}=\max \{u, 0\}$.

The main results of this article are as follows.

Theorem 2. Set $s \in(0,1), n>p s, K$ fulfilling condition (3), if $0<q<1,1<r<p_{s}^{*}$, then there exists $\lambda_{\star}>0$, such that for $\lambda$ $\in\left(0, \lambda_{\star}\right)$, equation (1) has at least two positive solutions.

Theorem 3. Set $s \in(0,1), n>p s$, and $\Omega$ be an open bounded domain in $\mathbb{R}^{n}$ with Lipschitz boundary. $K$ fulfilling the condition (3), if $0<q<1, r=p_{s}^{*}-1$, assumes that there exists $u_{0}$ $\in X_{0} \backslash\{0\}$ with $u_{0} \geq 0$ almost everywhere in $\mathbb{R}^{n}$, such that

$$
\sup _{t \geq 0} \mathscr{I}_{K, p_{s}^{*}}\left(t u_{0}\right)<\frac{s}{n} S_{K}^{n / p s}
$$


where $\mathscr{I}_{K, p_{s}^{*}}$ will be introduced in Section 2. Then, there exists $\lambda_{2}>0$, such that for $\lambda \in\left(0, \lambda_{2}\right)$, problem (1) admits least two solutions.

\section{Preliminaries}

We define the energy functional

$$
J_{\lambda}: X_{0} \longrightarrow \mathbb{R}
$$

associated to problem (1) as

$$
J_{\lambda}(u)=\mathscr{I}_{K, p}(u)-\mathscr{I}_{\lambda}(u),
$$

with

$$
\begin{aligned}
\mathscr{F}_{K, p}(u)= & \frac{1}{p} \int_{Q}|u(x)-u(y)|^{p} K(x-y) \mathrm{d} x \mathrm{~d} y \\
& -\frac{1}{r+1} \int_{\Omega} u_{+}^{r+1}(x) \mathrm{d} x \\
\mathscr{I}_{\lambda}(u)= & \frac{\lambda}{q+1} \int_{\Omega} u_{+}^{q+1}(x) \mathrm{d} x .
\end{aligned}
$$

We can see that $J_{\lambda} \in C^{1}\left(X_{0}, \mathbb{R}\right)$ and

$$
\begin{aligned}
\left\langle J_{\lambda}^{\prime}(u), \varphi\right\rangle_{X_{0}}= & \int_{Q}|u(x)-u(y)|^{p-2}(u(x)-u(y)) \\
& \times(\varphi(x)-\varphi(y)) K(x-y) d x d y \\
& -\lambda \int_{\Omega} u_{+}^{q}(x) \varphi(x) \mathrm{d} x-\int_{\Omega} u_{+}^{r}(x) \varphi(x) \mathrm{d} x,
\end{aligned}
$$

for any $\varphi \in X_{0}$.

Now, we give the Nehari manifold

$$
\mathcal{N}_{\lambda}=\left\{u \in X_{0} \backslash\{0\}:\left\langle J_{\lambda}^{\prime}(u), u\right\rangle=0\right\},
$$

where $\langle$,$\rangle denotes the duality between X_{0}$ and its dual space. Thus, $u \in \mathcal{N}_{\lambda}$ if and only if

$$
\|u\|_{X_{0}}^{p}-\lambda \int_{\Omega} u_{+}^{q+1}(x) \mathrm{d} x-\int_{\Omega} u_{+}^{r+1}(x) \mathrm{d} x=0 .
$$

The Nehari manifold $\mathcal{N}_{\lambda}$ is closely related to the following function $\varphi_{u}: t \mapsto J_{\lambda}(t u)$ for $t>0$ defined by

$$
\begin{aligned}
\varphi_{u}(t):= & J_{\lambda}(t u)=\frac{t^{p}}{p}\|u\|_{X_{0}}^{p_{0}}-\lambda \frac{t^{q+1}}{q+1} \int_{\Omega} u_{+}^{q+1}(x) \mathrm{d} x \\
& -\frac{t^{r+1}}{r+1} \int_{\Omega} u_{+}^{r+1}(x) \mathrm{d} x .
\end{aligned}
$$

Remark 4. Set $u \in X_{0} \backslash\{0\}$, then $t u \in \mathcal{N}_{\lambda}$ if and only if $\varphi_{u}^{\prime}(t)$ $=0$.
Moreover,

$\varphi_{u}^{\prime}(t)=t^{p-1}\|u\|_{X_{0}}^{p}-\lambda t^{q} \int_{\Omega} u_{+}^{q+1}(x) \mathrm{d} x-t^{r} \int_{\Omega} u_{+}^{r+1}(x) \mathrm{d} x$,

$$
\begin{aligned}
\varphi_{u}^{\prime}(t)= & (p-1) t^{p-2}\|u\|_{X_{0}}^{p}-q \lambda t^{q-1} \int_{\Omega} u_{+}^{q+1}(x) \mathrm{d} x \\
& -r t^{r-1} \int_{\Omega} u_{+}^{r+1}(x) \mathrm{d} x .
\end{aligned}
$$

According to (25) and Remark 4, for $u \in \mathcal{N}_{\lambda}$, we have

$$
\begin{aligned}
\varphi_{u}^{\prime}(1) & =(p-1)\|u\|_{X_{0}}^{p}-\lambda q \int u_{+}^{p+1}(x) \mathrm{d} x-r \int_{\Omega} u_{+}^{r+1}(x) \mathrm{d} x \\
& =(p-r-1) \int_{\Omega} u_{+}^{r+1}(x) \mathrm{d} x+\lambda(p-q-1) \int_{\Omega} u_{+}^{q+1}(x) \mathrm{d} x \\
& =(p-q-1)\|u\|_{X_{0}}^{p}-(q+r) \int_{\Omega} u_{+}^{r+1}(x) \mathrm{d} x \\
& =(p-r-1)\|u\|_{X_{0}}^{p}-\lambda(q-r) \int_{\Omega} u_{+}^{q+1}(x) \mathrm{d} x .
\end{aligned}
$$

The $\mathcal{N}_{\lambda}$ is divided into three sets, which are local minimum, local maximum, and local inflection point, respectively, i.e.,

$$
\begin{aligned}
& \mathcal{N}_{\lambda}^{+}=\left\{u \in \mathcal{N}_{\lambda}: \varphi_{u}^{\prime}(1)>0\right\}, \\
& \mathcal{N}_{\lambda}^{+}=\left\{u \in \mathcal{N}_{\lambda}: \varphi_{u}^{\prime}(1)>0\right\}, \\
& \mathcal{N}_{\lambda}^{0}=\left\{u \in \mathcal{N}_{\lambda}: \varphi_{u}^{\prime}(1)=0\right\} .
\end{aligned}
$$

To prove our result, we should start to show the following auxiliary lemmas.

Lemma 5. If $u_{0}$ is a local minimizer of $J_{\lambda}$ on $\mathcal{N}_{\lambda}$ and $u_{0} \notin \mathcal{N}_{\lambda}^{0}$, then $u_{0}$ is a critical point of $J_{\lambda}$.

Similar to Theorem 2.3 in [14], we can get this conclusion.

About fibering maps and the Nehari manifold, considering the function $\psi_{u}: \mathbb{R}^{+} \longrightarrow \mathbb{R}$ defined by

$$
\psi_{u}(t)=t^{1-q}\|u\|_{X_{0}}^{p}-t^{r-q} \int_{\Omega} u_{+}^{r+1} d x .
$$

Obviously, for any $t>0, t u \in \mathcal{N}_{\lambda}$ if and only if

$$
\psi_{u}(t)=\lambda \int_{\Omega} u_{+}^{q+1} d x .
$$

Moreover,

$$
\psi_{u}^{\prime}(t)=(1-q) t^{-q}\|u\|_{X_{0}}^{p}-(r-q) t^{r-q-1} \int_{\Omega} u_{+}^{r+1} d x
$$


and moreover, we know that $t u \in \mathcal{N}_{\lambda}$, then

$$
t^{q} \psi_{u}^{\prime}(t)=\varphi_{u}^{\prime}(t)
$$

So, $t u \in \mathcal{N}_{\lambda}^{+}\left(\operatorname{or} \mathcal{N}_{\lambda}^{-}\right)$if and only if $\psi_{u}^{\prime}(t)>0$ (or $\left.<0\right)$. Assume $u \in X_{0}$ and $u_{+} \neq 0$. In view of (29), $\psi_{u}$ fulfills the following properties:

(i) $\psi_{u}$ has a unique critical point at $t=t_{\max }(u)=$ $\left(\left((1-q)\|u\|_{X_{0}}^{p_{0}}\right) /\left((r-q) \int_{\Omega} u_{+}^{r+1} d x\right)\right)^{1 /(r-1)}>0$

(ii) $\psi_{u} \uparrow$ on $\left(0, t_{\max }(u)\right)$ and $\downarrow$ on $\left(t_{\max }(u),+\infty\right)$

$$
\lim _{t \longrightarrow+\infty} \psi_{u}(t)=-\infty
$$

Further, it follows from $\int_{\Omega} u_{+}^{q+1} d x>0$ that (30) has no solutions if $\lambda$ fulfills

$$
\begin{aligned}
\lambda \int_{\Omega} u_{+}^{q+1} d x> & \psi_{u}\left(t_{\max }(u)\right)=\left[\left(\frac{1-q}{r-q}\right)^{(1-q) /(r-1)}\right. \\
& \left.-\left(\frac{1-q}{r-q}\right)^{(r-q) /(r-1)}\right] \frac{\|u\|_{X_{0}}^{(p(r-q)) /(r-1)}}{\left(\int_{\Omega} u_{+}^{r+1} d x\right)^{(1-q) /(r-1)}} .
\end{aligned}
$$

According to (25) and (30) if $\lambda$ fulfills (34), then $\varphi_{u}^{\prime}(t)>0$. It seems $\varphi_{u}^{\prime}(t)<0$ as $\lambda$ is sufficiently large. Therefore, $t u \notin \mathcal{N}_{\lambda}$ for any $t>0$. Moreover, if $\lambda$ fulfills

$$
0<\lambda \int_{\Omega} u_{+}^{q+1} d x<\psi_{u}\left(t_{\max }(u)\right)
$$

then there exist $t_{1}$ and $t_{2}$ with $t_{1}<t_{\max }(u)<t_{2}$, such that

$$
\psi_{u}\left(t_{1}\right)=\psi_{u}\left(t_{2}\right)=\lambda \int_{\Omega} u_{+}^{q+1} d x, \text { and } \psi_{u}^{\prime}\left(t_{1}\right)>0, \psi_{u}^{\prime}\left(t_{2}\right)<0
$$

combining (25) and (30), which imply that $\varphi_{u}^{\prime}\left(t_{1}\right)=\varphi_{u}^{\prime}\left(t_{2}\right)=0$. It follows from (32) that $\varphi_{u}^{\prime}\left(t_{1}\right)>0, \varphi_{u}^{\prime}\left(t_{2}\right)<0$, which mean that the fibering map $\varphi_{u}$ admits a local minimum $t_{1} u \in \mathcal{N}_{\lambda}^{+}$ and a local maximum at $t_{2} u \in \mathcal{N}_{\lambda}^{-}$.

\section{The Subcritical Case: $0<q<1<r<p_{s}^{*}-1$}

Firstly, we prove the following lemmas.

Lemma 6. There exists $\lambda_{\star}>0$, such that for any $\lambda \in\left(0, \lambda_{\star}\right)$, we have $\mathcal{N}_{\lambda}^{0}=\varnothing$.
Proof. Using the inverse method, if $\mathcal{N}_{\lambda}^{0} \neq \varnothing$ for any $\lambda>0$. Then,

$$
\begin{aligned}
\left\langle J_{\lambda}^{\prime}(u), u\right\rangle_{X_{0}} & =0, \\
\varphi_{u}^{\prime}(1) & =0 .
\end{aligned}
$$

for $u \in \mathcal{N}_{\lambda}^{0}$.

Namely,

$$
\begin{aligned}
\|u\|_{X_{0}}^{p} & =\lambda \int_{\Omega} u_{+}^{q+1} \mathrm{~d} s+\int_{\Omega} u_{+}^{r+1} \mathrm{~d} x, \text { and }\|u\|_{X_{0}}^{2} \\
& =\lambda q \int_{\Omega} u_{+}^{q+1} \mathrm{~d} s+r \int_{\Omega} u_{+}^{r+1} \mathrm{~d} x
\end{aligned}
$$

Thus,

$$
\begin{aligned}
(1-q)\|u\|_{X_{0}}^{p} & =(r-q) \int_{\Omega} u_{+}^{r+1} \mathrm{~d} x, \text { and }(r-1)\|u\|_{X_{0}}^{p} \\
& =\lambda(r-q) \int_{\Omega} u_{+}^{q+1} \mathrm{~d} x .
\end{aligned}
$$

Using the Hölder inequality and Remark 4, there exist two positive constants $C_{1}, C_{2}$ such that

$$
\|u\|_{X_{0}}^{p} \leq C_{1}\|u\|_{X_{0}}^{r+1} \text { and }\|u\|_{X_{0}}^{p} \leq \lambda C_{2}\|u\|_{X_{0}}^{q+1}
$$

It yields that $C_{1}^{1 /(p-r-1)} \leq\|u\|_{X_{0}} \leq\left(\lambda C_{2}\right)^{1 /(p-q-1)}$. If $\lambda$ is small enough, then it is impossible. Thus, assuming no, the original set is empty.

Lemma 7. $J_{\lambda}$ is coercive and bounded from below on $\mathcal{N}_{\lambda}$ for $\lambda \in\left(0, \lambda_{\star}\right)$.

Proof. Let $u \in \mathcal{N}_{\lambda}$, (19) and (23) we get

$$
J_{\lambda}(u)=\left(\frac{1}{p}-\frac{1}{r+1}\right)\|u\|_{X_{0}}^{p}-\lambda\left(\frac{1}{q+1}-\frac{1}{r+1}\right) \int_{\Omega} u_{+}^{q+1} \mathrm{~d} x
$$

Using Remark 4 and Hölder inequality, we get

$$
\int_{\Omega} u_{+}^{q+1} \mathrm{~d} x \leq C_{n, q, s, \theta,|\Omega|}\|u\|_{X_{0}}^{q+1}
$$

Prove complete due to $0<q<1<r$.

By Lemmas 6 and 7 , for any $\lambda \in\left(0, \lambda_{\star}\right)$, we get $\mathcal{N}_{\lambda}=$ $\mathcal{N}_{\lambda}^{+} \cup \mathcal{N}_{\lambda}^{-}$, and so, $J_{\lambda}$ is coercive and bounded from below on $\mathcal{N}_{\lambda}^{+}$and $\mathcal{N}_{\lambda}^{-}$. Therefore, we define

$$
\alpha_{\lambda}^{+}=\inf _{u \in \mathcal{N}_{\lambda}^{+}} J_{\lambda}(u), \alpha_{\lambda}^{-}=\inf _{u \in \mathcal{N}_{\lambda}^{-}} J_{\lambda}(u)
$$

We have the following result. 
Proposition 8. If $0<\lambda<\lambda_{\star}$, then the functional $J_{\lambda}$ has a minimizer $u_{1}$ in $\mathcal{N}_{\lambda}^{+}$and satisfies

(1) $J_{\lambda}\left(u_{1}\right)=\inf _{u \in \mathcal{N}_{\lambda}^{+}} J_{\lambda}(u)<0$.

(2) $u_{1}$ is a solution of problem (1).

Proof. Since the bounded from below of $J_{\lambda}$ on $\mathcal{N}_{\lambda}^{+}$, there exists a minimizing sequence $\left\{u_{k}\right\} \subset \mathcal{N}_{\lambda}^{+}$, such that

$$
\lim _{k \rightarrow \infty} J_{\lambda}\left(u_{k}\right)=\inf _{u \in \mathcal{N}_{\lambda}^{+}} J_{\lambda}(u)
$$

We know that the sequence $\left\{u_{k}\right\}$ is bounded in $X_{0}$ by Lemma 7. $\left(X_{0},\|\cdot\|_{X_{0}}\right)$ is a Hilbert space (see Lemma 7 in [15]); thus, there exists $u_{1} \in X_{0}$ such that, up to a subsequence,

$$
\begin{gathered}
\int_{Q}\left|u_{k}(x)-u_{k}(y)\right|^{p-2}\left(u_{k}(x)-u_{k}(y)\right)(\phi(x)-\phi(y)) K(x-y) \mathrm{d} x \mathrm{~d} y \\
\longrightarrow \int_{Q}\left|u_{1}(x)-u_{2}(y)\right|^{p-2}\left(u_{1}(x)-u_{1}(y)\right) \\
\cdot(\phi(x)-\phi(y)) K(x-y) \mathrm{d} x \mathrm{~d} y \text { for } \forall \phi \in X_{0},
\end{gathered}
$$

when $k \longrightarrow \infty$. Further, by Lemma 8 in [15], we have

$$
u_{k} \longrightarrow u_{1} \text { in } L^{r}\left(\mathbb{R}^{n}\right), \quad u_{k} \longrightarrow u_{1} \text { a.e } \mathbb{R}^{n},
$$

as $k \longrightarrow \infty$, and by ([16], TheoremIV -9 ), there exists $\ell \epsilon$ $L^{r}\left(\mathbb{R}^{n}\right)$ such that

$$
\left|u_{k}(x)\right| \leq \ell(x) \text { a.e in } \mathbb{R}^{n}
$$

for any $1 \leq r<p_{s}^{*}=n p /(n-p s)(n>p s)$. It follows from the dominated convergence theorem that

$$
\begin{aligned}
\int_{\Omega}\left(u_{k}\right)_{+}^{q+1} \mathrm{~d} x & \longrightarrow \int_{\Omega}\left(u_{1}\right)_{+}^{q+1} \mathrm{~d} x, \text { and } \int_{\Omega}\left(u_{k}\right)_{+}^{r+1} \mathrm{~d} x \\
& \longrightarrow \int_{\Omega}\left(u_{1}\right)_{+}^{r+1} \mathrm{~d} x,
\end{aligned}
$$

ask $\longrightarrow \infty$. So, there exists $t_{1}$ such that $t_{1} u_{1} \in \mathcal{N}_{\lambda}^{+}$and $J_{\lambda}\left(t_{1} u_{1}\right)<0$. Therefore, we get $\inf _{u \in \mathcal{N}_{\lambda}^{+}} J_{\lambda}(u)<0$.

In order to prove that $u_{k} \longrightarrow u_{1}$ strongly in $X_{0}$. Still use the arc method if not, then $\left\|u_{1}\right\|_{X_{0}}<\liminf _{k \longrightarrow \infty}\left\|u_{k}\right\|_{X_{0}}$. Hence, for $\left\{u_{k}\right\} \in \mathcal{N}_{\lambda}^{+}$, we get

$$
\begin{aligned}
\lim _{k \longrightarrow \infty} \varphi_{u_{k}}^{\prime}\left(t_{1}\right)= & \lim _{k \longrightarrow \infty}\left(t_{1}\left\|u_{k}\right\|_{X_{0}}^{p}-\lambda t_{1}^{q} \int_{\Omega}\left(u_{k}\right)_{+}^{q+1} \mathrm{~d} x\right. \\
& \left.-t_{1}^{r} \int_{\Omega}\left(u_{k}\right)_{+}^{r+1} \mathrm{~d} x\right)>t_{1}\left\|u_{1}\right\|_{X_{0}}^{p} \\
& -\lambda t_{1}^{q} \int_{\Omega}\left(u_{1}\right)_{+}^{q+1} \mathrm{~d} x-t_{1}^{r} \int_{\Omega}\left(u_{1} x\right)_{+}^{r+1} \mathrm{~d} x \\
= & \varphi_{u_{1}}^{\prime}\left(t_{1}\right)=0 .
\end{aligned}
$$

That is, $\varphi_{u_{k}}^{\prime}\left(t_{1}\right)>0$ for $k$ large enough. Since $u_{k}=1, u_{k} \epsilon$ $\mathcal{N}_{\lambda}^{+}$, we infer that $\varphi_{u_{k}}^{\prime}(t)<0$ for $t \in(0,1)$ and $\varphi_{u_{k}}^{\prime}(1)=0$ for all $k$. So, must be $t_{1}>1$. In addition because $\varphi_{u_{1}}(t)$ is decreasing on $\left(0, t_{1}\right)$, and so,

$$
J_{\lambda}\left(t_{1} u_{1}\right) \leq J_{\lambda}\left(u_{1}\right)<\lim _{k \longrightarrow \infty} J_{\lambda}\left(u_{k}\right)=\inf _{u \in \mathcal{N}_{\lambda}^{+}} J_{\lambda}(u) .
$$

Obviously, the above equation is a contradiction. Therefore, $u_{k} \longrightarrow u_{1}$ strongly in $X_{0}$. It means that

$$
J_{\lambda}\left(u_{k}\right) \longrightarrow J_{\lambda}\left(u_{1}\right)=\inf _{u \in \mathcal{N}_{\lambda}^{+}} J_{\lambda}(u) \text { as } k \longrightarrow \infty,
$$

i.e., $u_{1}$ is a minimizer if $J_{\lambda}$ on $\mathcal{N}_{\lambda}^{+}$. By Lemma $5, u_{1}$ is a solution to problem (1).

Proposition 9. If $0<\lambda<\lambda_{1}$, then $J_{\lambda}$ admits a minimizer $u_{2}$ in $\mathscr{N}_{\lambda}^{-}$and satisfies

(1) $J_{\lambda}\left(u_{2}\right)=\inf _{u \in \mathcal{N}_{\lambda}^{-}} J_{\lambda}(u)>0$.

(2) $u_{2}$ is a solution to problem (1).

Proof. Since the bounded from below of $J_{\lambda}$ on $\mathcal{N}_{\lambda}^{-}$, there exists a minimizing sequence $\left\{\tilde{u}_{k}\right\} \subset \mathcal{N}_{\lambda}^{-}$, such that

$$
\lim _{k \longrightarrow \infty} J_{\lambda}\left(\tilde{u}_{k}\right)=\inf _{u \in \mathcal{N}_{\lambda}^{-}} J_{\lambda}(u)
$$

Similar to Proposition 8 , there exists $u_{2} \in X_{0}$ such that

$$
\begin{gathered}
\int_{Q}\left|\tilde{u}_{k}(x)-\tilde{u}_{k}(y)\right|^{p-2}\left(\tilde{u}_{k}(x)-\tilde{u}_{k}(y)\right)(\phi(x)-\phi(y)) K(x-y) \mathrm{d} x \mathrm{~d} y \\
\longrightarrow \int_{Q}\left|u_{2}(x)-u_{2}(y)\right|^{p-2}\left(u_{2}(x)-u_{2}(y)\right) \\
\cdot(\phi(x)-\phi(y)) K(x-y) \mathrm{d} x \mathrm{~d} y \text { for } \forall \phi \in X_{0},
\end{gathered}
$$

as $k \longrightarrow \infty$, and

$$
\begin{aligned}
& \int_{\Omega}\left(\tilde{u}_{k}\right)_{+}^{q+1} \mathrm{~d} x \longrightarrow \int_{\Omega}\left(u_{2}\right)_{+}^{q+1} \mathrm{~d} x, \text { and } \\
& \int_{\Omega}\left(\tilde{u}_{k}\right)_{+}^{r+1} \mathrm{~d} x \longrightarrow \int_{\Omega}\left(u_{2}\right)_{+}^{r+1} \mathrm{~d} x,
\end{aligned}
$$


as $k \longrightarrow \infty$. Moreover, from the nature of the fibering maps $\varphi_{u}(t)$, we infer that there exist $t_{1}, t_{2}$ with $t_{1}<t_{\max }(u)<t_{2}$ such that $t_{1} u \in \mathcal{N}_{\lambda}^{+}, t_{2} u \in \mathcal{N}_{\lambda}^{-}$, and $J_{\lambda}\left(t_{1} u\right) \leq J_{\lambda}(t u) \leq J_{\lambda}\left(t_{2} u\right)$.

Next, we show that $\tilde{u}_{k} \longrightarrow u_{2}$ strongly in $X_{0}$. If not, then $\left\|u_{2}\right\|_{X_{0}}<\lim \inf _{k \longrightarrow \infty}\left\|\tilde{u}_{k}\right\|_{X_{0}}$. Thus, for $\left\{\tilde{u}_{k}\right\} \in \mathcal{N}_{\lambda}^{-}$, we have $J_{\lambda}\left(\tilde{u}_{k}\right) \geq J_{\lambda}\left(t \tilde{u}_{k}\right)$ for all $t \geq t_{\max }(u)$, and

$$
\begin{aligned}
J_{\lambda}\left(t_{2} u_{2}\right)= & \frac{t_{2}^{p}}{p}\left\|u_{2}\right\|_{X_{0}}^{p}-\lambda \frac{t_{2}^{q+1}}{q+1} \int_{\Omega}\left(u_{2}\right)_{+}^{q+1} \mathrm{~d} x-\frac{t_{2}^{r+1}}{r+1} \int_{\Omega}\left(u_{2}\right)_{+}^{r+1} \mathrm{~d} x \\
< & \lim _{k \rightarrow \infty}\left(\frac{t_{2}^{p}}{p}\left\|\tilde{u}_{k}\right\|_{X_{0}}^{p}-\lambda \frac{t_{2}^{q+1}}{q+1} \int_{\Omega}\left(\tilde{u}_{k}\right)_{+}^{q+1} \mathrm{~d} x\right. \\
& \left.-\frac{t_{2}^{r+1}}{r+1} \int_{\Omega}\left(\tilde{u}_{k}\right)_{+}^{r+1} \mathrm{~d} x\right)=\lim _{k \longrightarrow \infty} J_{\lambda}\left(t_{2} \tilde{u}_{k}\right) \\
\leq & J_{\lambda}\left(\tilde{u}_{k}\right)=\inf _{u \in \mathcal{N}_{\lambda}^{-}} J_{\lambda}(u),
\end{aligned}
$$

in a similar way, we still can get a contradiction. Thus, $\tilde{u}_{k}$ $\longrightarrow u_{2}$ strongly in $X_{0}$. It means that

$$
J_{\lambda}\left(\tilde{u}_{k}\right) \longrightarrow J_{\lambda}\left(u_{2}\right)=\inf _{u \in \mathcal{N}_{\lambda}^{-}} J_{\lambda}(u) \text { as } k \longrightarrow \infty
$$

Namely, $u_{2}$ is a minimizer if $J_{\lambda}$ on $\mathcal{N}_{\lambda}^{-} \cdot u_{2}$ is a solution to problem (1) according to Lemma 5.

Proof of Theorem 10. We obtain that problem (1) has two solutions $u_{1} \in \mathcal{N}_{\lambda}^{+}$and $u_{2} \in \mathcal{N}_{\lambda}^{-}$in $X_{0}$ due to the Propositions 8 and 9 and Lemma 5; moreover, we know that two solutions are distinct since $\mathcal{N}_{\lambda}^{+} \cap \mathcal{N}_{\lambda}^{-}=\varnothing$.

\section{The Critical Case: $0<q<1, r=p_{s}^{*}-1$}

For the critical case, since the embedding $X_{0} \hookrightarrow L^{p_{s}^{*}}(\Omega)$ is not compact, then the energy functional does not satisfy the Palais-Smale condition globally, but it is true for the energy functional in a suitable range related to the best fractional critical Sobolev constant in the embedding $X_{0} \hookrightarrow L^{p_{s}^{*}}\left(\mathbb{R}^{n}\right)$. For this, we define fractional Sobolev best constant $S_{K}$ as

$S_{K}=\inf _{v \in X_{0} \backslash\{0\}} \frac{\int_{\mathbb{R}^{2 n}}|v(x)-v(y)|^{p} K(x-y) \mathrm{d} x \mathrm{~d} y}{\left(\int_{\Omega}|v(x)|^{p_{s}^{*}}\right)^{p / p_{s}^{*}}}$ for $v \in X_{0} \backslash\{0\}$.

Before we give the Proof of Theorem 13, we start by some auxiliary results. Firstly, using the same proofs of Lemma 6 , we deduce that there exists $\lambda_{*}>0$ such that $\mathscr{N}_{\lambda}^{0}$ $=\varnothing$ for each $\lambda \in\left(0, \lambda_{*}\right)$. Also, it is clear that $J_{\lambda}$ is coercive and bounded from below on $\mathcal{N}_{\lambda}$ for $\lambda \in\left(0, \lambda_{*}\right)$ by Lemma 7. So, for any $\lambda \in\left(0, \lambda_{*}\right)$, we also obtain that $\mathcal{N}_{\lambda}=\mathcal{N}_{\lambda}^{+} \cup$
$\mathcal{N}_{\lambda}^{-}$, and $J_{\lambda}$ is coercive and bounded from below on $\mathcal{N}_{\lambda}^{+}$ and $\mathcal{N}_{\lambda}^{-}$. We define

$$
\tilde{\alpha}_{\lambda}^{+}=\inf _{u \in \mathcal{N}_{\lambda}^{+}} J_{\lambda}(u), \tilde{\alpha}_{\lambda}^{-}=\inf _{u \in \mathcal{N}_{\lambda}^{-}} J_{\lambda}(u)
$$

Proposition 11. Let $\left\{u_{k}\right\} \subset X_{0}$ be a $(P S)_{c}$ sequence for $J_{\lambda}$ with

$$
c<\frac{s}{n} S_{K}^{n / p s}-M \lambda^{p_{s}^{*} / p_{s}^{\star}-q},
$$

then there exists a subsequence of $\left\{u_{k}\right\}$, which converges strongly in $X_{0}$, where $S_{K}$ is defined in (57) and $M>0$ is defined by

$$
\begin{aligned}
M= & \frac{(2 n-(n-2 s)(q+1))(1-q)}{4(q+1)} \\
& \cdot\left(\frac{(1-q)(n-2 s)}{4 s}\right)^{(q+1) /\left(p_{s}^{\star}-(q+1)\right)}|\Omega| .
\end{aligned}
$$

Proof. It follows from $\left\{u_{k}\right\}$ is bounded in $X_{0}$ that there exists $u_{\infty} \in X_{0}$ such that $u_{k} \longrightarrow u_{\infty}$ weakly in $X_{0}$, that is

$$
\begin{gathered}
\int_{Q}\left|u_{k}(x)-u_{k}(y)\right|^{p-2}\left(u_{k}(x)-u_{k}(y)\right)(\phi(x)-\phi(y)) K(x-y) \mathrm{d} x \mathrm{~d} y \\
\longrightarrow \int_{Q}\left|u_{\infty}(x)-u_{\infty}(y)\right|^{p-2}\left(u_{\infty}(x)-u_{\infty}(y)\right) \\
\cdot(\phi(x)-\phi(y)) K(x-y) \mathrm{d} x \mathrm{~d} y \text { for } \forall \phi \in X_{0},
\end{gathered}
$$

as $k \longrightarrow \infty$. Moreover, using the same arguments as lemma 9 ([17]), we get that

$$
\begin{aligned}
& u_{k} \longrightarrow u_{\infty} \text { weakly in } L^{p_{s}^{*}}\left(\mathbb{R}^{n}\right) ; \\
& u_{k} \longrightarrow u_{\infty} \text { in } L^{r}\left(\mathbb{R}^{n}\right) ; \\
& u_{k} \longrightarrow u_{\infty} \text { a.e.in } \mathbb{R}^{n}
\end{aligned}
$$

as $k \longrightarrow \infty$, and by ([16], TheoremIV -9], there exists $\ell \in$ $L^{r}\left(\mathbb{R}^{n}\right)$ such that

$$
\left|u_{k}(x)\right| \leq \ell(x) \text { a.e.in } \mathbb{R}^{n},
$$

for any $1 \leq r<p_{s}^{*}=n p /(n-p s)(n>p s)$. Then, using dominated convergence theorem, we have that

$$
\int_{\Omega}\left(u_{k}\right)_{+}^{q+1} \mathrm{~d} x \longrightarrow \int_{\Omega}\left(u_{\infty}\right)_{+}^{q+1} \mathrm{~d} x
$$

Also, by the same method as in ([18], Lemma 1.32), we get 


$$
\begin{aligned}
& \int_{Q}\left|u_{k}(x)-u_{k}(y)\right|^{p} K(x-y) \mathrm{d} x \mathrm{~d} y \\
& \longrightarrow \int_{Q}\left|u_{k}(x)-u_{\infty}(x)-u_{k}(y)+u_{\infty}(y)\right|^{p} K(x-y) \mathrm{d} x \mathrm{~d} y \\
& +\int_{Q}\left|u_{\infty}(x)-u_{\infty}(y)\right|^{p} K(x-y) \mathrm{d} x \mathrm{~d} y+o(1), \\
& \int_{\Omega}\left(u_{k}(x)\right)_{+}^{p_{s}^{*}} \mathrm{~d} x=\int_{\Omega}\left(\left(u_{k}-u_{\infty}\right)(x)\right)_{+}^{p_{s}^{*}} \mathrm{~d} x \\
& +\int_{\Omega}\left(u_{\infty}(x)\right)_{+}^{p_{s}^{*}} \mathrm{~d} x+o(1),
\end{aligned}
$$

as $k \longrightarrow \infty$. Then,

$$
\begin{aligned}
\left\langle J_{\lambda}^{\prime}\left(u_{k}\right), u_{k}\right\rangle_{X_{0}}= & \int_{Q}\left|u_{k}(x)-u_{k}(y)\right|^{p} K(x-y) \mathrm{d} x \mathrm{~d} y \\
& -\lambda \int\left(u_{k}(x)\right)_{+}^{q+1} \mathrm{~d} x-\int_{\Omega}\left(u_{k}(x)\right)_{+}^{p_{s}^{*}} \mathrm{~d} x \\
= & \int_{\Omega} \mid u_{k}(x)-u_{\infty}(x)-u_{k}(y) \\
& +\left.u_{\infty}(y)\right|^{p} K(x-y) \mathrm{d} x \mathrm{~d} y \\
& +\int_{Q}\left|u_{\infty}(x)-u_{\infty}(y)\right|^{p} K(x-y) \mathrm{d} x \mathrm{~d} y \\
& -\lambda \int_{\Omega}\left(u_{k}(x)\right)_{+}^{q+1} \mathrm{~d} x-\left(\int _ { \Omega } \left(\left(u_{k}-u_{\infty}\right)\right.\right. \\
& \left.\cdot(x))_{+}^{p_{s}^{*}} \mathrm{~d} x+\int_{\Omega}\left(u_{\infty}\right)_{+}^{r} \mathrm{~d} x+o(1)\right)+o(1) \\
= & \int_{Q}\left|\left(u_{k}-u_{\infty}\right)(x)-\left(u_{k}-u_{\infty}\right)(y)\right|^{p} \\
& \cdot K(x-y) \mathrm{d} x \mathrm{~d} y-\int_{\Omega}\left(\left(u_{k}-u_{\infty}\right)(x)\right)_{+}^{p_{s}^{*}} \mathrm{~d} x \\
& +\left\langle J_{\lambda}^{\prime}\left(u_{\infty}\right), u_{\infty}\right\rangle X_{0}+o(1) .
\end{aligned}
$$

By $\left\langle J_{\lambda}^{\prime}\left(u_{\infty}\right), u_{\infty}\right\rangle_{X_{0}}=0$ and $\left\langle J_{\lambda}^{\prime}\left(u_{k}\right), u_{k}\right\rangle_{X_{0}} \longrightarrow 0$ as $k$ $\longrightarrow \infty$, we know that

$$
\begin{array}{rl}
\left\|u_{k}-u_{\infty}\right\|_{X_{0}}^{p}= & \int_{Q}\left|\left(u_{k}-u_{\infty}\right)(x)-\left(u_{k}-u_{\infty}\right)(y)\right|^{p} \\
\cdot K(x-y) \mathrm{d} x \mathrm{~d} y \longrightarrow b & b \\
\int_{\Omega}\left(\left(u_{k}-u_{\infty}\right)(x)\right)_{+}^{p_{s}^{*}} \mathrm{~d} x \longrightarrow b, \quad \text { as } k \longrightarrow \infty
\end{array}
$$

If $b=0$, is clearly true. If $b>0$, in view of the definition of $S_{K}$ in 17 , we get

$$
\left\|u_{k}-u_{\infty}\right\|_{X_{0}}^{p} \geq S_{K}\left(\int_{\Omega}\left(\left(u_{k}-u_{\infty}\right)(x)\right)_{+}^{p_{s}^{*}} \mathrm{~d} x\right)^{p / p_{s}^{2}} .
$$

Thus, we have $b \geq S_{K} b^{p / p_{s}^{*}}$. That is, $b \geq S_{K}^{n / p s}$. On the other hand, we have

$$
\begin{aligned}
c= & \lim _{k \rightarrow \infty} J_{\lambda}\left(u_{k}\right)=\lim _{k \longrightarrow \infty}\left(\frac{1}{p}\left\|u_{k}\right\|_{X_{0}}^{p}-\lambda \frac{1}{q+1} \int_{\Omega}\left(u_{k}(x)\right)_{+}^{q+1} \mathrm{~d} x\right. \\
& \left.-\frac{1}{r+1} \int_{\Omega}\left(u_{k}(x)\right)_{+}^{p_{\star}} \mathrm{d} x\right) \geq J_{\lambda}\left(u_{\infty}\right)+\frac{s}{n} S_{K}^{n / p s} .
\end{aligned}
$$

By the assumption that $c<(s / n) S_{K}^{n / p s}$, we have $J_{\lambda}\left(u_{\infty}\right)$ $<0$. In particular, $u_{\infty} \neq 0$ and

$0<\frac{1}{p}\left\|u_{\infty}\right\|_{X_{0}}^{p}<\frac{1}{p_{s}^{*}} \int_{\Omega}\left(u_{\infty}(x)\right)_{+}^{p_{s}^{*}} \mathrm{~d} x+\lambda \frac{1}{q+1} \int_{\Omega}\left(u_{\infty}(x)\right)_{+}^{q+1} \mathrm{~d} x$.

Then,

$$
\begin{aligned}
c= & \lim _{k \longrightarrow \infty} J_{\lambda}\left(u_{k}\right)=\lim _{k \longrightarrow \infty}\left(J_{\lambda}\left(u_{k}\right)-\frac{1}{p}\left\langle J_{\lambda}^{\prime}\left(u_{k}\right), u_{k}\right\rangle_{X_{0}}\right) \\
= & \lim _{k \longrightarrow \infty}\left(\frac{s}{n} \int_{\Omega}\left(\left(u_{\mathrm{k}}-u_{\infty}\right)(x)\right)_{+}^{p_{s}^{*}} \mathrm{~d} x+\frac{s}{n} \int_{\Omega}\left(u_{\infty}(x)\right)_{+}^{p_{s}^{*}} \mathrm{~d} x\right. \\
& \left.+\lambda\left(\frac{1}{p}-\frac{1}{q+1}\right) \int_{\Omega}\left(u_{k}(x)\right)_{+}^{q+1} \mathrm{~d} x\right) \\
= & \frac{s}{n} b+\frac{s}{n} \int_{\Omega}\left(u_{\infty}(x)\right)_{+}^{p_{s}^{*}} \mathrm{~d} x+\lambda\left(\frac{1}{p}-\frac{1}{q+1}\right) \int_{\Omega}\left(u_{\infty}(x)\right)_{+}^{q+1} \mathrm{~d} x \\
\geq & \frac{s}{n} S_{K}^{n / p s}+\frac{s}{n} \int_{\Omega}\left(u_{\infty}(x)\right)_{+}^{p^{*}} \mathrm{~d} x+\lambda\left(\frac{1}{p}-\frac{1}{q+1}\right) \int_{\Omega}\left(u_{\infty}(x)\right)_{+}^{q+1} \mathrm{~d} x .
\end{aligned}
$$

Moreover, by Hölder inequality, we have

$$
\int_{\Omega}\left(u_{\infty}(x)\right)_{+}^{q+1} \mathrm{~d} x \leq|\Omega|^{\left(p_{s}^{*}-(q+1)\right) / p_{s}^{*}}\left(\int_{\Omega}\left(u_{\infty}(x)\right)_{+}^{p_{s}^{*}} \mathrm{~d} x\right)^{(q+1) / p_{s}^{*}} .
$$

Thus,

$$
\begin{aligned}
c \geq & \frac{s}{n} S_{K}^{n / p s}+\frac{s}{n}\left(\int_{\Omega}\left(u_{\infty}(x)\right)_{+}^{p_{s}^{*}} \mathrm{~d} x\right) \\
& +\lambda\left(\frac{1}{p}-\frac{1}{q+1}\right)|\Omega|^{\left(p_{s}^{*}-(q+1)\right) / p_{s}^{*}} \\
& \cdot\left(\int_{\Omega}\left(u_{\infty}(x)\right)_{+}^{p_{s}^{*}} \mathrm{~d} x\right)^{(q+1) / p_{s}}:=\frac{s}{n} S_{K}^{n / p s}+h(\eta),
\end{aligned}
$$

where

$$
\begin{aligned}
h(\eta) & =\frac{s}{n} \eta^{p_{s}^{*}}+\lambda\left(\frac{1}{p}-\frac{1}{q+1}\right)|\Omega|^{\left(p_{s}^{*}-(q+1)\right) / p_{s}^{*}} \eta^{q+1} \text { with } \eta \\
& =\left(\int_{\Omega}\left(u_{\infty}(x)\right)_{+}^{p_{s}^{*}} \mathrm{~d} x\right)^{1 / p_{s}^{*}} .
\end{aligned}
$$


So, $h(\eta)$ attains its minimum at $\eta_{0}=(\lambda(p-q-1)$ $(n-p s) / 2 p s)^{1 /\left(p_{s}^{\star}-(q+1)\right)}|\Omega|^{1 / p_{s}^{\star}}$ and

$$
\begin{aligned}
h\left(\eta_{0}\right)= & -\frac{(2 n-(n-p s)(q+1))(p-q-1)}{2 p(q+1)} \\
& \cdot\left(\frac{(p-1-q)(n-2 s)}{2 p s}\right)^{(q+1) /\left(p_{s}^{\star}-(q+1)\right)}|\Omega| \lambda^{p_{s}^{*} /\left(p_{s}^{*}-(q+1)\right)} \\
= & -M \lambda^{p_{s}^{*} / p_{s}^{*}-(q+1)} .
\end{aligned}
$$

Therefore,

$$
c \geq \frac{s}{n} S_{K}^{n / p s}-M \lambda^{p_{s}^{*} /\left(p_{s}^{*}-(q+1)\right)},
$$

which is a contradiction. Therefore, $b=0$ and we obtain that $u_{k} \longrightarrow u_{\infty}$ strongly in $X_{0}$.

Proposition 12. There exists $\lambda_{2}>0$ and $u_{0} \in X_{0}$ such that

$$
\sup _{t>0} J_{\lambda}\left(t u_{0}\right)<\frac{s}{n} S_{K}^{n / p s}-M \lambda^{p_{s}^{*} /\left(p_{s}^{*}-(q+1)\right)}
$$

for $\lambda \in\left(0, \lambda_{2}\right)$. In particular

$$
\tilde{\alpha}_{\lambda}^{-}<\frac{s}{n} S_{K}^{n / p s}-M \lambda^{p_{s}^{*} /\left(p_{s}^{*}-(q+1)\right)} .
$$

Proof. We suppose there exists $\lambda_{* *}>0$ such that $(s / n) S_{K}^{n / p s}$ $-M \lambda^{p_{s}^{*} /\left(p_{s}^{\star}-(q+1)\right)}>0$ for all $\lambda \in\left(0, \lambda_{* *}\right)$. By condition (17) we have that there is $u_{0} \in X_{0} \backslash\{0\}$ such that

$$
\begin{aligned}
J_{\lambda}\left(t u_{0}\right) & \leq \sup _{t \geq 0} \mathscr{I}_{K, p_{s}^{*}}\left(t u_{0}\right)-\lambda \frac{t^{q+1}}{q+1} \int_{\Omega}\left(u_{0}\right)_{+}^{q+1} \mathrm{~d} x \\
& <\frac{s}{n} S_{K}^{n / p s}-\lambda \frac{t_{0}^{q+1}}{q+1} \int_{\Omega}\left(u_{0}\right)_{+}^{q+1} \mathrm{~d} x .
\end{aligned}
$$

Let $\lambda_{* * *}:=\left(t_{0}^{q+1} \int_{\Omega}\left(u_{0}\right)_{+}^{q+1} \mathrm{~d} x /(M(q+1))\right)^{\left(p_{s}^{*}-(q+1)\right) /(q+1)}$. Therefore, for $\lambda \in\left(0, \lambda_{* * *}\right)$, we obtain that

$$
-\frac{t_{0}^{q+1}}{q+1} \lambda \int_{\Omega}\left(u_{0}\right)_{+}^{q+1} \mathrm{~d} x<-M \lambda^{p_{s}^{*} /\left(p_{s}^{*}-(q+1)\right)} .
$$

Then, we have (77) holds.

Finally, let $\lambda_{2}=\min \left\{\lambda_{*}, \lambda_{* *}, \lambda_{* * *}\right\}$, we obtain that

$$
\tilde{\alpha}_{\lambda}^{-}<\frac{s}{n} S_{K}^{n / p s}-M \lambda^{p_{s}^{*} /\left(p_{s}^{*}-(q+1)\right)},
$$

for $\lambda \in\left(0, \lambda_{2}\right)$ by the nature of fibering maps $\varphi_{u}(t)=J_{\lambda}(t u)$.
Proof of Theorem 13. There exist two sequences $\left\{u_{k}^{+}\right\}$and $\left\{u_{k}^{-}\right\}$in $X_{0}$ such that

$J_{\lambda}\left(u_{k}^{+}\right) \longrightarrow \tilde{\alpha}_{\lambda}^{+}, J_{\lambda}^{\prime}\left(u_{k}^{+}\right) \longrightarrow 0$ and $J_{\lambda}\left(u_{k}^{-}\right) \longrightarrow \tilde{\alpha}_{\lambda}^{-}, J_{\lambda}^{\prime}\left(u_{k}^{-}\right) \longrightarrow 0$,

as $k \longrightarrow \infty$ because of Propositions 11 and 12. From related properties of fibering maps $\varphi_{u}(t)$, we have $\tilde{\alpha}_{\lambda}^{+}<0$. Similar to the Proof of Theorem 10, problem (1) admits two solutions $\tilde{u}_{1} \in \mathcal{N}_{\lambda}^{+}$and $\tilde{u}_{2} \in \mathcal{N}_{1}^{-}$in $X_{0}$. Moreover, these two solutions are distinct since $\mathcal{N}_{\lambda}^{+} \cap \mathcal{N}_{\lambda}^{-}=\varnothing$.

\section{Conflicts of Interest}

The authors declare that they have no competing interests.

\section{References}

[1] M. G. Crandall, P. H. Rabinowitz, and L. Tartar, "On a Dirichlet problem with a singular nonlinearity," Communications in Partial Differential Equations, vol. 2, pp. 193-222, 1977.

[2] Y. Haitao, "Multiplicity and asymptotic behavior of positive solutions for a singular semilinear elliptic problem," Journal of Differential Equations, vol. 189, no. 2, pp. 487-512, 2003.

[3] J. Hernandez, J. Francisco, and J. M. Vega, "Positive solutions for singular nonlinear elliptic equations," Proceedings of the Royal Society of Edinburgh, vol. 137, no. 1, pp. 41-62, 2007.

[4] J. Hernandez, J. Kartson, and P. L. Simon, "Multiplicity for semilinear elliptic equations involving singular nonlinearity," Nonlinear Analysis, vol. 65, no. 2, pp. 265-283, 2006.

[5] N. Hirano, C. Saccon, and N. Shioji, "Brezis-Nirenberg type theorems and multiplicity of positive solutions for a singular elliptic problem," Journal of Differential Equations, vol. 245, no. 8, pp. 1997-2037, 2008.

[6] N. Hirano, C. Saccon, and N. Shioji, "Existence of multiple positive solutions for singular elliptic problems with concave and convex nonlinearities," Advances in Differential Equations, vol. 9, no. 1-2, pp. 197-220, 2004.

[7] T. Mukherjee and K. Sreenadh, "Critical growth fractional elliptic problem with singular nonlinearities," Electronic Journal of Differential Equations, vol. 54, pp. 1-23, 2016.

[8] J. Zuo, M. Li, B. Li, and Z. Qiao, "A bifurcation and symmetry result for critical fractional Laplacian equations involving a perturbation," Advances in Difference Equations, vol. 2020, Article ID 58, 2020.

[9] J. Zuo, T. An, G. Ye, and Z. Qiao, "Nonhomogeneous fractional $p$-Kirchhoff problems involving a critical nonlinearity," Electronic Journal of Qualitative Theory of Differential Equations, vol. 41, pp. 1-5, 2019.

[10] B. Ge, Y.-X. Cui, L.-L. Sun, and M. Ferrara, "The positive solutions to a quasi-linear problem of fractional $p$-Laplacian type without the Ambrosetti-Rabinowitz condition," Positivity, vol. 22, no. 3, pp. 873-895, 2018.

[11] J. Zuo, T. An, and M. Li, "Superlinear Kirchhof-type problems of the fractional $p$-Laplacian without the (AR) condition," Boundary Value Problems, vol. 2018, Article ID 180, 2018.

[12] J. Zuo, T. An, L. Yang, and X. Ren, “The Nehari manifold for a fractional p-Kirchhoff system involving sign- changing weight function and concave-convex nonlinearities," Journal of Function Spaces, vol. 2019, 9 pages, 2019. 
[13] M. Xiang, B. Zhang, and M. Freeara, "Existence of solutions for Kirchhoff type problem involving the non-local fractional $p$ Laplacian," Journal of Mathematical Analysis and Applications, vol. 424, no. 2, pp. 1021-1041, 2015.

[14] K. J. Brown and Y. Zhang, "The Nehari manifold for a semilinear elliptic equation with a sign-changing weight function," Journal of Difference Equations, vol. 193, no. 2, p. 499, 2003.

[15] R. Servadei and E. Valdinoci, "Mountain pass solutions for non-local elliptic operators," Journal of Mathematical Analysis and Applications, vol. 389, no. 2, pp. 887-898, 2012.

[16] H. Brezis, Analyse Fonctionelle. Thorie et applications, Masson, Paris, 1983.

[17] R. Servadei and E. Valdinoci, "The Brezis-Nirenberg result for the fractional Laplacian," Transactions of the American Mathematical Society, vol. 367, pp. 67-102, 2015.

[18] M. Willem, Minimax Theorems, Birkhäuser, Boston, 1996. 